\author{
Achim H. Kaim \\ Rolf Hügli \\ Harald M. Bonél \\ Gernot Jundt
}

\section{Chondroblastoma and clear cell chondrosarcoma: radiological and MRI characteristics with histopathological correlation}

Received: 12 June 2001

Accepted: 15 October 2001

Published online: 14 December 2001

(C) ISS 2001

\author{
A.H. Kaim ( $) \cdot$ R. Hügli \\ Institute of Diagnostic Radiology, \\ University Hospital Basle, Petersgraben 4, \\ 4031 Basle, Switzerland \\ e-mail: akaim@uhbs.ch \\ Tel.: +41-61-2654344 \\ Fax: +41-61-2655383 \\ H.M. Bonél \\ Institute of Clinical Radiology, \\ University Hospital Munich-Grosshadern, \\ Germany \\ G. Jundt \\ Institute of Pathology, \\ University Hospital Basle, \\ Basle, Switzerland
}

\author{
Abstract Objective: To analyze and \\ compare the radiological and magnetic \\ resonance imaging (MRI) appearances \\ of chondroblastoma and clear cell \\ chondrosarcoma with histopathologi- \\ cal correlation. Design and patients: \\ Twelve patients with histologically \\ proven chondroblastoma and of an- \\ other four patients with clear cell \\ chondrosarcoma were investigated by \\ radiographs and MRI (T1-, T2- \\ weighted sequences, intravenous ga- \\ dolinium application). Additionally, \\ the clinical and radiologic data of \\ seven cases of clear cell chondrosar- \\ coma without available MRI were \\ considered. The localization, calcifi- \\ cation of tumor matrix, periosteal re- \\ action, cortical bone and patterns of \\ bone destruction were analyzed ac- \\ cording to the Lodwick radiological \\ grading system (LRGS). The signal \\ intensity on T1- and T2-weighted se- \\ quences, characteristics of contrast \\ enhancement, associated bone mar- \\ row edema, soft tissue reaction and \\ joint involvement were evaluated. \\ Histopathological specimens were \\ available in all cases. Results: The \\ age of patients with chondroblastoma \\ (range 15-59 years, mean 22.3 years) \\ was lower than that of those with \\ clear cell chondrosarcoma (range \\ 19-61 years, mean 36.6 years), and \\ the lesions were smaller in the chon- \\ droblastoma group (range 1-4 cm, \\ mean $2.3 \mathrm{~cm}$ ) than in patients with \\ clear cell chondrosarcoma (range \\ $3-7.5 \mathrm{~cm}$, mean $5.2 \mathrm{~cm}$ ). The chon-
}

droblastomas were more confined to the epiphysis (10/12) than the clear cell chondrosarcomas. All chondroblastomas and clear cell chondrosarcomas except one were classified as grade $1 \mathrm{~A}$ or $1 \mathrm{~B}$ according to the LRGS; one clear cell chondrosarcoma was judged as grade 2. Signal intensity of the tumors on MRI was very heterogeneous in both groups. High signal intensity on T2-weighted MR images in chondroblastoma mostly corresponded to areas with aneurysmal bone cyst components and in clear cell chondrosarcoma to islands of hyaline cartilage. Contrast enhancement occurred in all tumors and tended to be more intense with clear cell chondrosarcoma. Chondroblastoma was more frequently associated with bone marrow edema (11/12), periosteal reaction (10/12), soft tissue reaction (7/12) and synovitis (3/12). Conclusion: Chondroblastoma occurs in younger patients, is smaller than clear cell chondrosarcoma and is more confined to the epiphysis. The overlap of signal intensity and contrast enhancement patterns does not allow a reliable differentiation of the two tumors by MRI. Chondroblastomas are typically associated with bone marrow edema, periosteal reaction and soft tissue reaction.

Keywords Chondroblastoma $\cdot$ Clear cell chondrosarcoma $\cdot$ Magnetic resonance imaging $\cdot$ MRI . Histopathology 


\section{Introduction}

Chondroblastoma is a rare, benign cartilaginous bone tumor that is treated by curettage, and overall has a good prognosis with a low morbidity. The tumor appears at centers of enchondral ossification, most commonly in the epiphysis of the long bones. Very rarely the epicenter may be in the metaphysis or apophysis. Radiographically it is a sharply delineated osteolytic defect with a sclerotic border. Subtle cartilaginous calcifications are seen in over $50 \%$ of cases and a periosteal reaction may also be present $[1,2]$. The differential diagnoses of a tumor localized in the epiphysis are limited, but include osteomyelitis, giant cell tumor, and clear cell chondrosarcoma. Giant cell tumors can mostly be differentiated by the age of the patient, the size of the lesion, its extension into the metaphysis and its poorer delineation. Clear cell chondrosarcoma and chondroblastoma, however, may have a very similar radiographic appearance, which prevents a reliable differentiation of the two tumors. The mean age of tumor occurrence in clear cell chondrosarcoma is one decade later, but the overlap of the age range complicates the differential diagnosis. A precise diagnosis is important as the therapy of clear cell chondrosarcoma is more aggressive than that of chondroblastoma and may involve en-bloc resection $[3,4,5]$.

Previous studies of the MRI appearance of chondroblastoma have demonstrated that it is frequently associated with bone marrow edema, periosteal and soft tissue reactions and varying signal intensity on T2-weighted sequences $[6,7,8]$. It seems doubtful whether the MRI criteria as described in previous case reports [9] allow a reliable differentiation between chondroblastoma and the histologically malignant clear cell chondrosarcoma, which requires a more extended surgical approach and has an overall poorer prognosis.

The aim of this study was to compare the radiological and MRI patterns of the two tumors and to correlate specific radiological findings with histopathology.

\section{Patients and methods}

\section{Patients}

Between 1971 and 1999 a total of 93 cases of chondroblastoma and 11 cases of clear cell chondrosarcoma were referred to the Bone Tumor Reference Center for analysis. A retrospective analysis was performed by two experienced musculoskeletal radiologists (A.H.K., R.H.) and divided into one group (group 1) consisting of 12 cases of histologically proven chondroblastoma in which a diagnostic MRI examination was available, and a second group (group 2) comprising 11 patients with clear cell chondrosarcoma only four of whom had an MRI examination.

Group 1 consisted of 11 men and one woman, mean age 22.3 years (range 15-59 years). Seven lesions were located in the distal femur, two in the proximal tibia, and one each in the metacarpal bone of the first digit, the first metatarsal bone and the proximal humerus. In all the patients conventional radiographs and an MRI examination were obtained. Imaging was done prior to any diagnostic or therapeutic intervention. Group 2 comprised 11 patients ( 9 men, 2 women), median age 36.6 years (range 19-61 years) in whom the tumor was histologically diagnosed as clear cell chondrosarcoma. Conventional radiographs were obtained in all patients, but MR images in only four. Five lesions were located in the proximal femur, two in the proximal humerus, and one each in the distal femur, proximal tibia, upper pubic rim and maxilla.

\section{Imaging}

The MRI parameters varied depending on the institution performing the imaging, and the field strength ranged from 0.5 to $1.5 \mathrm{~T}$. T1- and T2-weighted spin echo or fast spin echo images were acquired in all patients with gadolinium-DTPA (standard intravenous dose of $0.1 \mathrm{mmol} / \mathrm{kg}$ body weight) enhanced T1-weighted sequences, four of which had spectral fat saturation. The lesions were documented in at least two orthogonal planes; the slice thickness varied between 3 and $6 \mathrm{~mm}$ according to the size of the lesion. The in-plane resolution was $1 \mathrm{~mm}^{2}$ or less for all MRI examinations. All examinations of this retrospective evaluation were performed for clinical indications.

\section{Analysis criteria}

Radiographs and MRI were analyzed by two radiologists (A.H.K., R.H.) in consensus. They were masked to the clinical and histological findings.

The radiographs were analyzed with respect to the anatomical location of the tumor, size (long axis size on radiographs), matrix calcification, periosteal reaction and tumor extent applying the Lodwick classification $[10,11]$.

The MRI features evaluated were signal intensity on T1- and T2- weighted images, morphology of the lesion itself (homogeneity, heterogeneity), contrast medium uptake (intense/ medium/ low, heterogeneous/ homogeneous), periosteal reaction, bone marrow edema, soft tissue reaction and synovitis with joint effusion. Lesions with a higher signal intensity on the T1- and T2-weighted sequences compared with fatty bone marrow were considered as hyperintense, and lesions with lower signal intensity were defined as hypointense.

\section{Histology}

All histological samples were analyzed by the same experienced musculoskeletal pathologist (G.J.). As all the chondroblastomas were treated by curettage and therefore no en-bloc resection was available, a general correlation with the radiological result was performed. Three of four chondrosarcomas that had been imaged with MRI were treated by en-bloc-resection and their histomorphology could be correlated with the MR study, whereas in one case only a diagnostic biopsy of the tumor was available that allowed a rather general correlation.

\section{Results}

Seven patients with chondroblastoma had a fully fused growth plate and in the remaining five patients it was partially fused. All 11 patients with clear cell chondrosarcoma had completely fused growth plates and apophyses. 
Fig. 1A-D Chondroblastoma of the first metatarsal bone in a 24-year-old male patient.

A Radiography depicts an expansile, sharply delineated lesion (Lodwick grade 1B; black arrowhead). B Sagittal, fatsuppressed, T2-weighted MR image depicts a lesion with a solid and a macrocystic component. C Sagittal T1-weighted MR image before (above) and after (below)intravenous gadolinium application shows moderate and homogeneous enhancement of the solid portion. D Pseudoepithelial lining of the aneurysmal bone cyst component (black arrows). Typical matrix calcification and chondroblastic tumor cells (hematoxylin-eosin (HE), $\times 10$ )
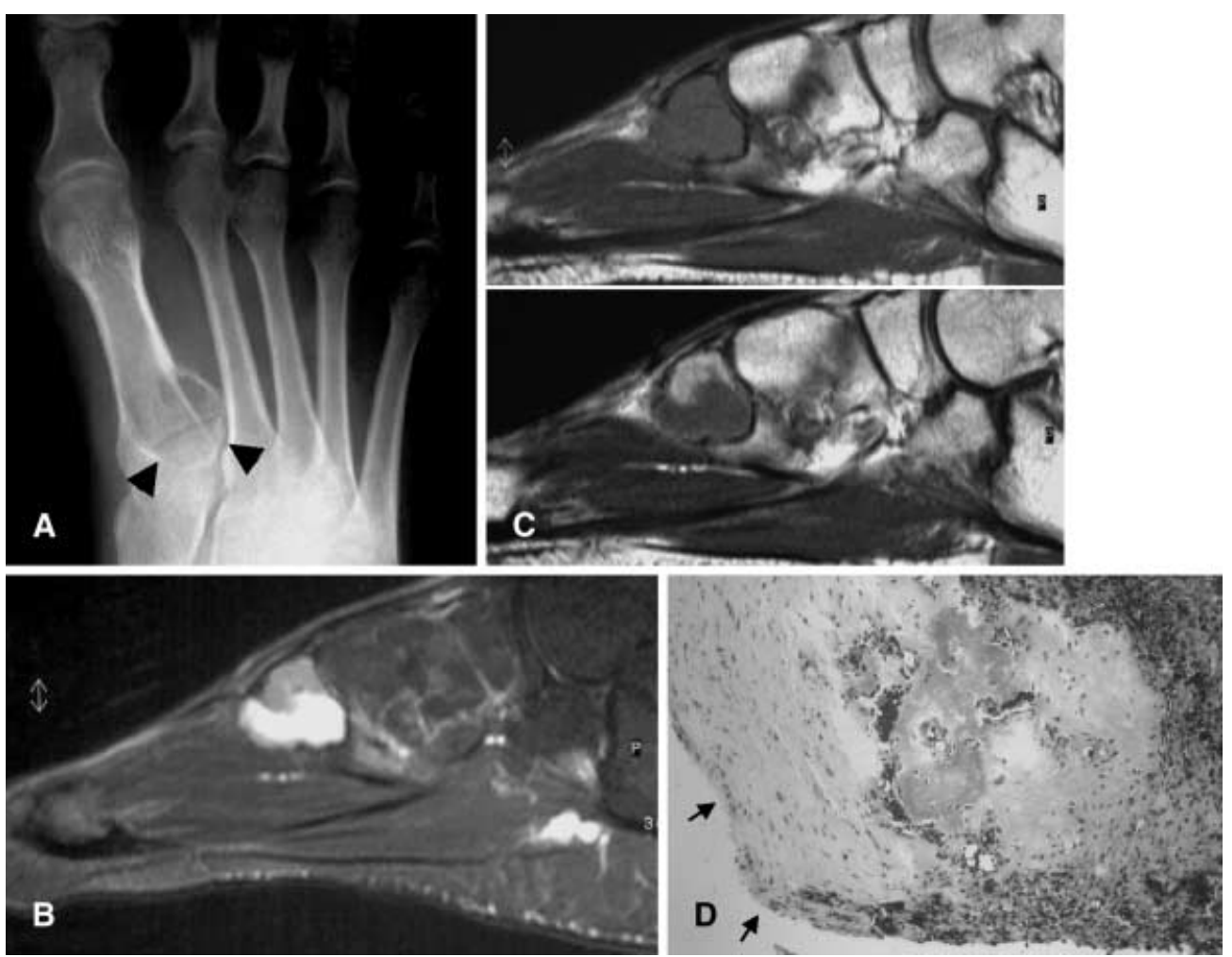

\section{Radiography}

\section{Chondroblastoma}

Two chondroblastomas were strictly confined to the epiphysis without demonstrable contact with the growth plate; eight were contiguous with the growth plate, of which two extended across the growth plate and involved the metaphysis. They ranged from 1 to $4 \mathrm{~cm}$ (mean $2.3 \mathrm{~cm}$ ) in size. Radiographically all lesions showed a geographic pattern, were sharply delineated and in nine a sclerotic border could also be detected (Figs. 1A, 2A, 3A). In three cases small lesions in the distal femoral epiphysis were difficult to appreciate on the radiographs without knowledge of the MRI findings.

In seven of 12 patients the chondroblastoma extended from the bone marrow to the cortical bone, resulting in expansion of the outer thinned cortical surface by more than $1 \mathrm{~cm}$ in four lesions (Fig. 1A). Seven of 12 lesions were Lodwick grade 1A and five were grade 1B. Stippled matrix calcification could be detected on the radiographs in only two cases. Periosteal reaction was seen in eight of 12 patients, and rated as laminar in six and interrupted in two lesions.

\section{Clear cell chondrosarcoma}

The locations of the clear cell chondrosarcomas $(n=11)$ were more widespread. Five lesions were epimetaphy- seal, two were metadiaphyseal (Fig. 4A), two were epiphyseal with extension into the growth plate and two lesions were in the axial skeleton (pubic and maxillary bones). The size varied from 3 to $7.5 \mathrm{~cm}$ (mean $5.2 \mathrm{~cm}$ ). In one case the tumor margins could not be clearly delineated because of permeative tumor.

Ten of 11 clear cell chondrosarcomas demonstrated geographic lesions, of which four were Lodwick grade $1 \mathrm{~B}$ and six were Lodwick grade $1 \mathrm{~A}$ lesions. One tumor revealed permeative destruction of the adjacent bony structures with disruption of the cortical border and was classified as grade 2 .

The cortical bone was thinned in four of 11 tumors and solid (Fig. 5A) or stippled matrix calcification was seen in six. A continuous periosteal reaction was observed in four tumors.

\section{MRI}

\section{Chondroblastoma}

MRI of the chondroblastomas showed that the signal intensity on the T1-weighted images was homogeneous hypointense in all 12 cases (Figs. 1C, 2C). The signal on $\mathrm{T} 2$-weighted sequences was mainly heterogeneous and six of 12 tumors were hypointense while six of 12 showed a heterogeneous pattern with partial hyper- and hypointense signal intensity. 
Fig. 2A-D Chondroblastoma of the epiphysis of the right distal femur in a 20 -year-old male patient. A Anteroposterior radiograph shows an expansile lesion (Lodwick grade 1A) with cortical thinning (black arrows). B T1-weighted MR image before (above) and after (below) intravenous gadolinium application depicts an inhomogeneously and moderately enhancing mass with concomitant bone marrow edema pattern, periosteal and soft tissue reaction. $\mathbf{C}$ T2-weighted MR image reveals several foci with hyperintense signal intensity corresponding to aneurysmal bone cyst components. D Aneurysmal bone cyst component in chondroblastoma $(\mathrm{HE}, \times 5)$
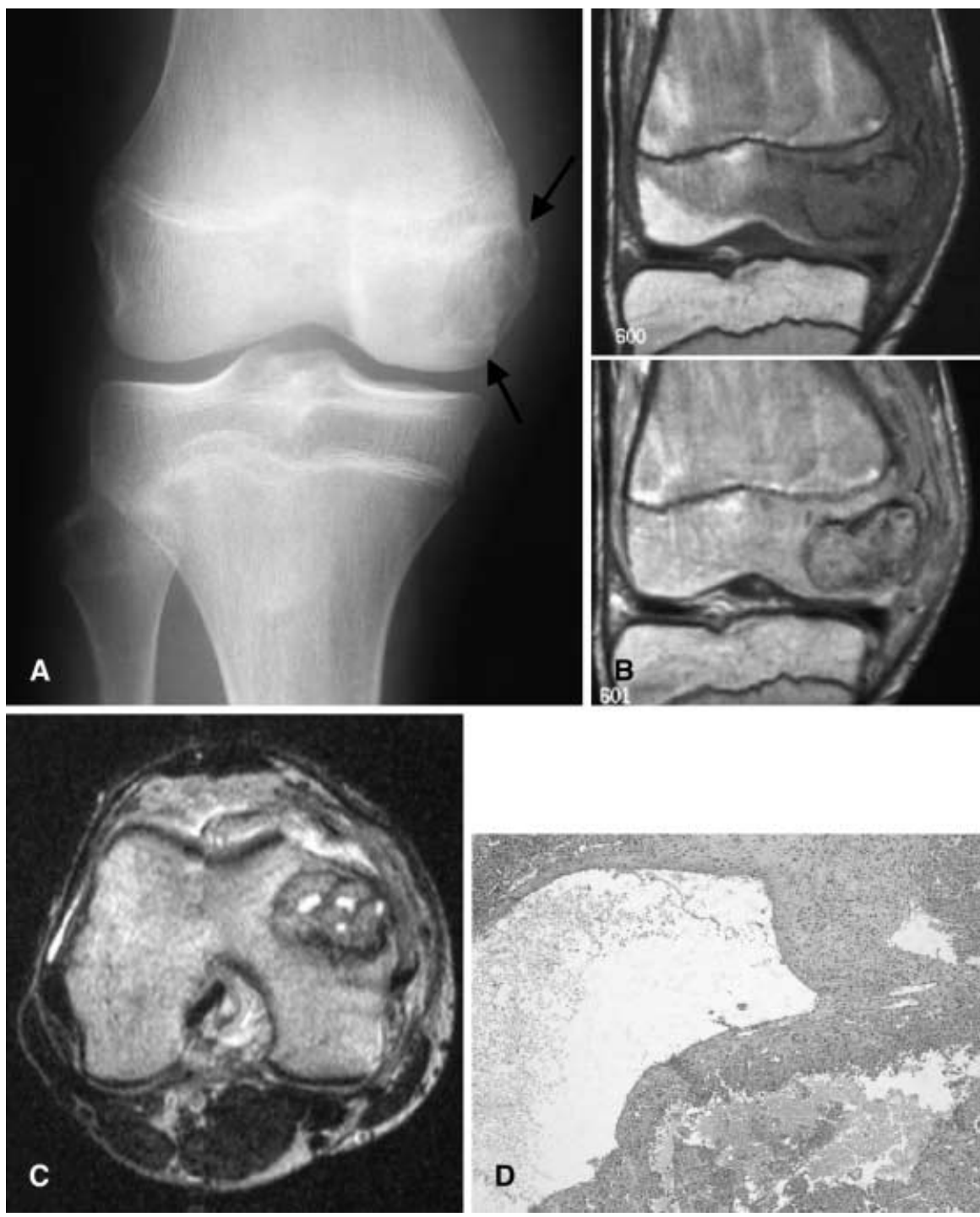

Two of the 6 patients with heterogeneous signal revealed 1-2 mm circumscribed zones of hyperintense signal intensity in otherwise hypodense areas. The histological analysis of these patients showed multiple small hemorrhagic areas within the cell-enriched tumor as well as sinusoid blood-filled spaces, which might explain the subtle signal alterations.

In four of 6 patients macrocystic components could be detected on MRI, corresponding to parts of an aneurysmal bone cyst histologically (Figs. 1B, D, 2B, D). The patients with hypointense signal intensity all demonstrated a cell-enriched tumor with chondroblastic tumor cells embedded in a chondroid matrix.

Heterogeneous and moderate enhancement in the central solid portion of the tumor (Figs. 1C, 2C) could be detected after contrast medium administration in nine of 12 cases. Three of 12 cases showed homogeneous and marked enhancement of the tumor (Fig. 3B).
Eleven of 12 patients revealed distinct adjacent peritumoral bone marrow edema (Figs. 2B, 3B) which could be differentiated from the tumor itself. The bone marrow edema was characterized by a poorly delineated area of homogeneous hyperintensity on T2-weighted sequences and diffuse enhancement after intravenous contrast medium. In 10 of 12 patients a periosteal reaction could be delineated on the T2-weighted and enhanced T1-weighted sequences (Figs. 2B, 3B). An adjacent soft tissue reaction with high signal intensity on $\mathrm{T} 2$-weighted sequences and contrast enhancement was detectable in seven of 12 cases (Fig. 2B). Joint effusion with concomitant synovial enhancement was found in three of 12 cases.

\section{Clear cell chondrosarcoma}

In the clear cell chondrosarcoma group three patients (3/4) showed a heterogeneous pattern on the T2-weighted 

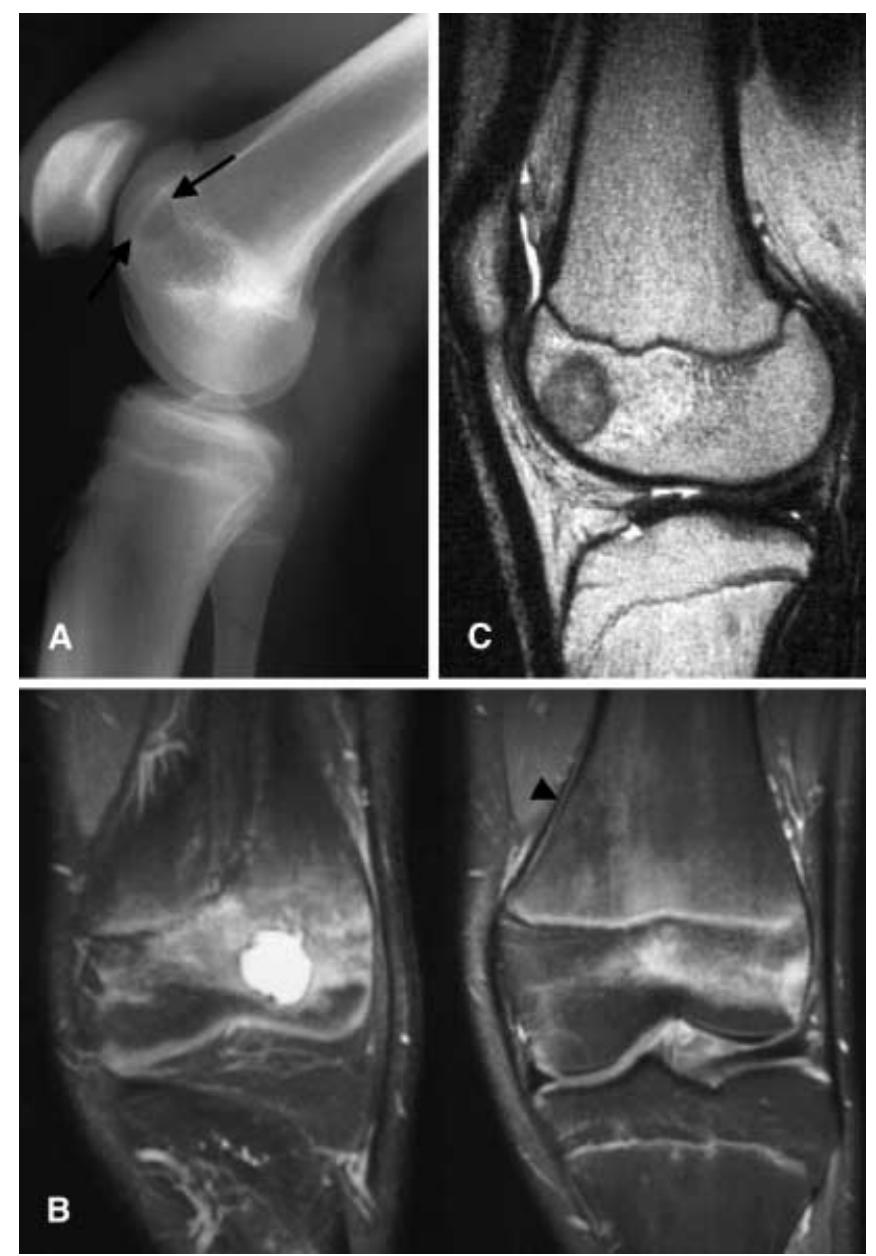

Fig. 3A-C A 15-year-old male patient with chondroblastoma of the left-sided distal femur. A Lateral radiograph reveals a lytic and epiphyseal lesion (Lodwick grade 1B; black arrows). B Fat-suppressed T1-weighted MR image after intravenous gadolinium shows a strongly enhancing mass with adjacent bone marrow edema and marked periosteal reaction (black arrowhead). C T2weighted MR image shows a hypointense lesion

sequences with overall low to intermediate signal intensity and several areas of high signal intensity $(2-4 \mathrm{~mm})$. In two patients parts of a conventional chondrosarcoma and islands with hyaline cartilage matrix could be delineated histologically (Fig. 4C, D). In one patient only a biopsy was obtainable so that an exact histomorphological diagnosis was not possible. On the T1-weighted sequences, however, the tumor was generally of low signal intensity. But three of four tumors revealed an inhomogeneity on the T1-weighted sequences with even less signal intensity in the central areas. After contrast medium administration all three lesions demonstrated heterogeneous uptake, the remaining hypodense regions corresponded to large areas of matrix calcifications (Fig. 4B).

One lesion (1/4) was homogeneous hypointense on the T1- and T2-weighted sequences, having a bulky area of calcified matrix on the radiograph (Fig. 5A-C). Histologically it was characterized by a cell-rich tumor with intense ossification of the cartilaginous tumor matrix (Fig. 5D). On the T1-weighted sequence a homogeneous hypointense pattern and heterogeneous, moderate contrast enhancement was demonstrated. Two of four tumors revealed neither an adjacent bone marrow edema nor a soft tissue reaction. One of four tumors was large, aggressive and permeative with concomitant bone marrow and periosteal reaction. Histological analysis in this patient showed, in addition to the clear cell chondrosarcoma, parts of a conventional grade 1 chondrosarcoma.

\section{Discussion}

Chondroblastoma has an incidence of less than $1 \%$ of all primary bone tumors, while clear cell chondrosarcoma is even rarer, accounting for only $2 \%$ of all chondrosarcomas [12]. Whereas chondroblastoma is a curable benign bone tumor with a low morbidity, clear cell chondrosarcoma is a low-grade malignant tumor, but with a better prognosis than conventional chondrosarcoma. Bjornsson et al. [5] found a mortality of $15 \%(n=7)$ in a series of 47 patients with clear cell chondrosarcoma with hematogenous dissemination present in all seven, of whom five had had inadequate initial surgical treatment (curettage 4 , circumscribed resection 1 ). The histological and cytological characteristics reveal a broad spectrum, and specialist knowledge of the pathologist is obligatory in order to reach a correct final diagnosis.

Chondroblastoma consists of immature chondrocytes, chondroblasts, and encompasses chondral or osteochondral matrix, which may calcify in a reticular pattern. Giant cells are also found, but histological differentiation from giant cell tumor is possible due to the presence of cartilaginous matrix and chondroblasts. In rare uncertain cases immunohistochemical analysis showing cells positive for S-100 protein will prove the chondral origin of these cells [13]. Between $20 \%$ and $30 \%$ of chondroblastomas are associated with elements of aneurysmal bone cysts [14]. This was the case in four of the 12 patients in our series. Chondroblastomas commonly present in the second decade, with $95 \%$ of the tumors being detected between the ages of 5 and 25 years, and the majority of the patients are male (male:female ratio 2:1) [12].

Clear cell chondrosarcoma is characterized histologically by large neoplastic cells with clear and slightly eosinophilic cytoplasm. The tumor can contain mixed features of a conventional chondrosarcoma $[4,5,15]$. Small areas of chondroid matrix with circumscribed calcifications may be seen, but osteoclastic giant cells can also be delineated relatively often, and tumor-induced osteoid formation may occur. The age range is relatively wide, with the majority presenting in the third and fourth 
Fig. 4 A 32-year-old patient with a metadiaphyseal clear cell chondrosarcoma. A Lateral radiograph shows a lytic, expansile lesion with matrix calcifications. B T1-weighted MR image before (left) and after (right) gadolinium application shows moderate enhancement with central hypointensities corresponding to calcifications. C T2-weighted MR image shows focally hyperintense areas. D Areas of a conventional chondrosarcoma grade 1 with hyaline cartilage matrix and calcifications (left) are in close contact with the cellenriched clear cell chondrosarcoma area $($ right $)(\mathrm{HE}, \times 5)$
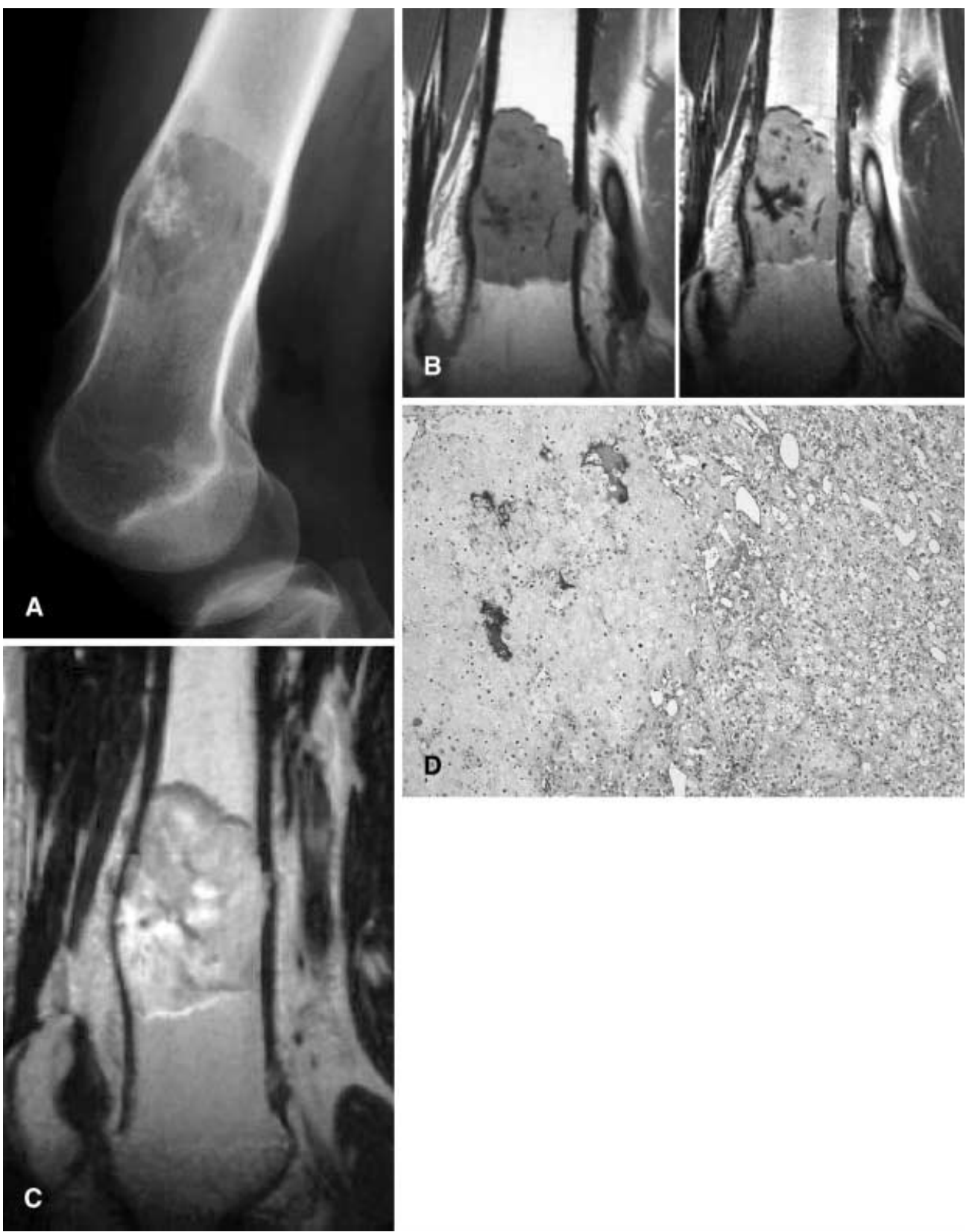

decades; there is a 2:1 male:female ratio [12]. Our series also reflected this distribution.

Both tumors may present as sharply delineated osteolytic lesions with stippled matrix calcification in the proximal or distal end of long bones. In our series the clear cell chondrosarcomas were larger than the chondroblastomas and overall more expansive. The chondroblastomas were confined to the epiphysis, whereas more than half the cases of clear cell chondrosarcoma extended into the metaphysis or the epicenter of the lesion was epimetaphyseal. All but one of the lesions were strictly geographic and sharply delineated, defined as Lodwick grade $1 \mathrm{~A}$ or $1 \mathrm{~B}$; the remaining clear cell chondrosarcoma was rated as grade 2 with permeative growth. On histological examination of this case a larger area of conventional grade 1 chondrosarcoma was found beside areas with the typical clear cells.

A feature of chondroblastomas on MRI was the extensive peritumoral, inflammation-related bone marrow edema, which was frequently associated with a soft tissue and a periosteal reaction. The latter was more frequently demonstrated on MRI compared with radiographs. These appearances can be confusing, although the mere presence and quantity of marrow and soft tissue 
Fig. 5A-D A 31-year-old patient with epiphyseal clear cell chondrosarcoma of the proximal tibial bone. a Lateral radiograph shows a sharply delineated sclerotic lesion (Lodwick grade $1 \mathrm{~A}$ ) of the proximal tibia (black arrow). B T1-weighted MR image before (left) and after (right) gadolinium application shows slight and heterogeneous contrast enhancement. C T2-weighted image shows a homogeneously hypointense tumor (black arrow). D Enlarged round tumor cells with clear to eosinophilic cytoplasm and centrally located mediumsized nuclei accompanied by osteoclasts. There is peripheral ossification of the cartilaginous tumor matrix $(\mathrm{HE}, \times 20)$
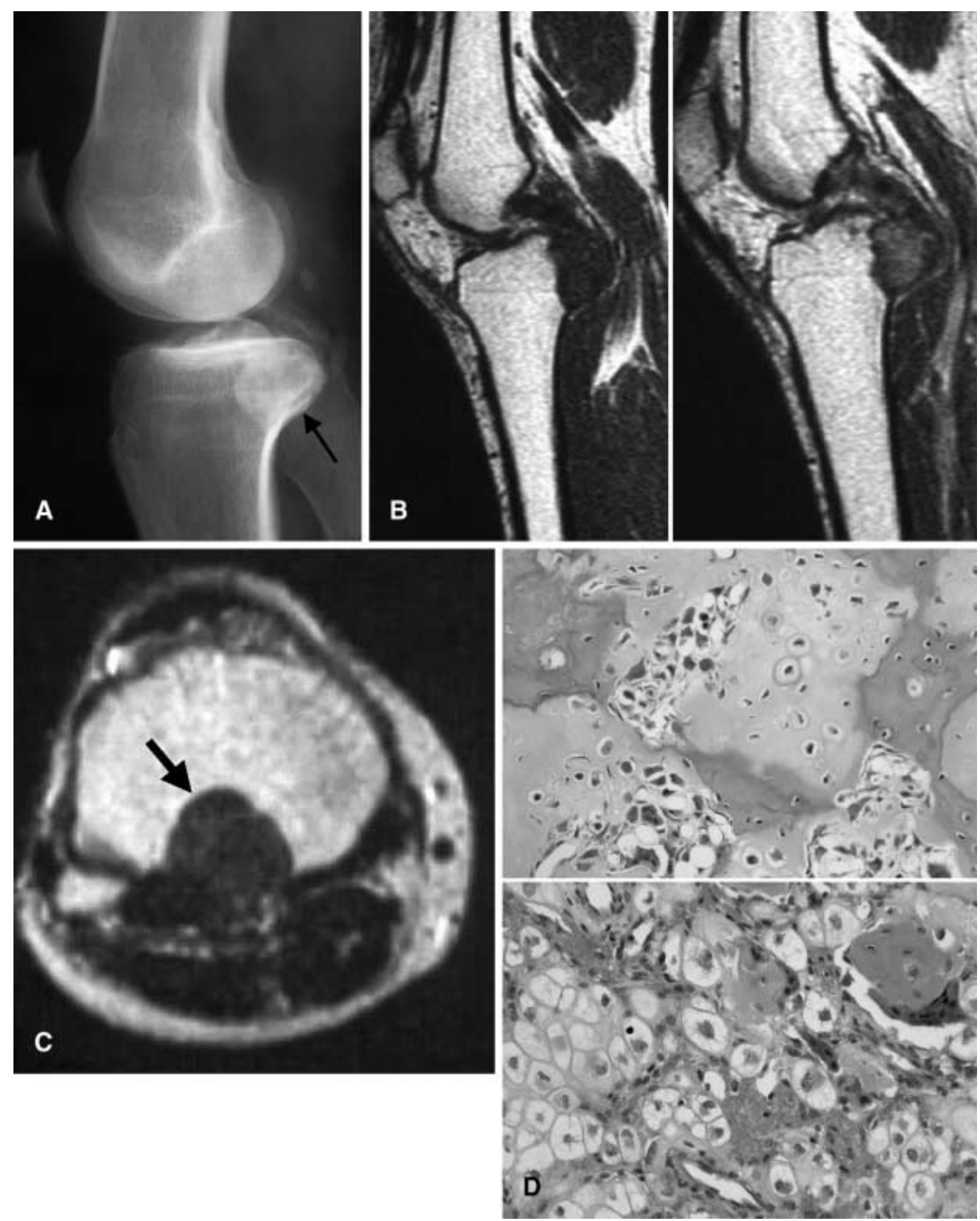

edema are unreliable indicators of the biological potential of a lesion [16]. Features indicative of tumor-associated bone marrow edema are frequently found in benign disorders such as osteoid osteoma, osteoblastoma and chondroblastoma $[16,17,18,19,20]$. Weatherall et al. [7] found 19 of 22 patients with chondroblastoma had bone marrow edema and a periosteal reaction was demonstrated in 18 patients, while Jee et al. [6] reported 82\% with similar features. Brower et al. [2] found periosteal reaction in $47 \%$ of chondroblastomas on radiographs, which is a significantly lower percentage in comparison with the MRI findings. The reason for this extensive concomitant reaction is unclear, but it is unlikely to be explained simply as a stress reaction. Possible mechanisms generating edema surrounding tumors have been described [21] and the edema represents a nonspecific response of tissue to a stimulus. Histological examination of these areas revealed edematous fluid together with inflammatory cells $[18,19]$, but this inflammatory response was not invariably found in other series [16].

It has been suggested that the signal intensity of these tumors on T2-weighted images is a differential feature to distinguish between chondroblastoma and clear cell chondrosarcoma $[9,22]$, but in our study both lesions showed heterogeneity. Chondroblastoma may have a homogeneous low signal intensity on T2-weighted se- 
quences but may also have circumscribed areas of higher signal intensity, corresponding to areas of aneurysmal bone cyst. Conversely, one clear cell chondrosarcoma demonstrated homogeneous low signal intensity on the T2-weighted images due to extensive tumor-induced secondary bone formation. Two tumors showed an inhomogeneous pattern with areas of hyaline matrix, corresponding to the regions of high signal intensity on MRI.

There was greater contrast enhancement in clear cell chondrosarcomas compared with chondroblastomas, but overlap occurs and this did not provide a clear differential diagnostic criterion. The contrast enhancement was heterogeneous in all clear cell chondrosarcomas and a similar pattern was present in three chondroblastomas.

\section{Conclusion}

Chondroblastoma occurs in a younger patient group than clear cell chondrosarcoma and is generally smaller and more confined to the epiphysis. There is overlap of signal intensity and contrast enhancement patterns with clear cell chondrosarcoma which precludes reliable differentiation of the two types of tumor by MRI. Chondroblastomas are typically associated with inflammationrelated peritumoral bone marrow edema, periosteal and soft tissue reactions, which may also occur in clear cell chondrosarcoma.

\section{References}

1. Bloem JL, Mulder JD. Chondroblastoma: a clinical and radiological study of 104 cases. Skeletal Radiol 1985; 14:1-9.

2. Brower AC, Moser RP, Kransdorf MJ. The frequency and diagnostic significance of periostitis in chondroblastoma. AJR Am J Roentgenol 1990; 154:309-314.

3. Gilbert TJ, Goswitz JJ, Griffiths H. Radiologic case study. Clear-cell chondrosarcoma. Orthopedics 1995; 18:407, 410-412.

4. Taconis WK. Clear cell chondrosarcoma: report of three cases and review of the literature. Diagn Imaging Clin Med 1986; 55:219-227.

5. Bjornsson J, Unni KK, Dahlin DC, Beabout JW, Sim FH. Clear cell chondrosarcoma of bone: observations in 47 cases. Am J Surg Pathol 1984; $8: 223-230$

6. Jee WH, Park YK, McCauley TR, et al. Chondroblastoma: MR characteristics with pathologic correlation. J Comput Assist Tomogr 1999; 23:721-726.

7. Weatherall PT, Maale GE, Mendelsohn DB, Sherry CS, Erdman WE, Pascoe HR. Chondroblastoma: classic and confusing appearance at MR imaging. Radiology 1994; 190:467-474.

8. Yamamura S, Sato K, Sugiura H, Iwata $\mathrm{H}$. Inflammatory reaction in chondroblastoma. Skeletal Radiol 1996; 25:371-376.
9. Fobben ES, Dalinka MK, Schiebler ML, et al. The magnetic resonance imaging appearance at 1.5 tesla of cartilaginous tumors involving the epiphysis. Skeletal Radiol 1987; 16:647-651.

10. Lodwick GS, Wilson AJ, Farrell C, Virtama P, Smeltzer FM, Dittrich F. Estimating rate of growth in bone lesions: observer performance and error. Radiology 1980; 134:585-590.

11. Lodwick GS, Wilson AJ, Farrell C, Virtama P, Dittrich F. Determining growth rates of focal lesions of bone from radiographs. Radiology 1980; 134:577-583

12. Fechner RE, Mills EM. Cartilaginous lesions. In: Fechner RE, Mills EM, eds. Tumors of the bones and joints, 3rd edn, vol 8. Washington, DC: Armed Forces Institute of Pathology, 1993.

13. Kurt AM, Unni KK, Sim FH, McLeod RA. Chondroblastoma of bone. Hum Pathol 1989; 20:965-976.

14. Springfield DS, Capanna R, Gherlinzoni F, Picci P, Campanacci M. Chondroblastoma: a review of seventy cases. J Bone Joint Surg Am 1985; 67:748-755.

15. Aigner T, Dertinger S, Belke J, Kirchner T. Chondrocytic cell differentiation in clear cell chondrosarcoma. Hum Pathol 1996; 27: 1301-1305.

16. Kroon HM, Bloem JL, Holscher HC, van der Woude HJ, Reijnierse M, Taminiau AH. MR imaging of edema accompanying benign and malignant bone tumors. Skeletal Radiol 1994; 23:261-269.
17. Biebuyck JC, Katz LD, McCauley T. Soft tissue edema in osteoid osteoma. Skeletal Radiol 1993; 22:37-41.

18. Yeager BA, Schiebler ML, Wertheim $\mathrm{SB}$, et al. MR imaging of osteoid osteoma of the talus. J Comput Assist Tomogr 1987; 11:916-917.

19. Crim JR, Mirra JM, Eckardt JJ, Seeger LL. Widespread inflammatory response to osteoblastoma: the flare phenomenon. Radiology 1990; 177:835-836.

20. Yamamura S, Sato K, Sugiura H, Asano M, Takahashi M, Iwata H. Magnetic resonance imaging of inflammatory reaction in osteoid osteoma. Arch Orthop Trauma Surg 1994; 114:8-13.

21. Steen RG. Edema and tumor perfusion: characterization by quantitative $1 \mathrm{H}$ MR imaging. AJR Am J Roentgenol 1992; 158:259-264.

22. Cohen EK, Kressel HY, Frank TS, et al. Hyaline cartilage-origin bone and soft-tissue neoplasms: MR appearance and histologic correlation. Radiology 1988; 167:477-481. 